\title{
Status of the Standard Model Prediction of the Muon g-2
}

\author{
M. Passera ${ }^{\text {a* }}$ \\ a Dipartimento di Fisica, Università di Padova and INFN, Via Marzolo 8, 35131 Padova, Italy
}

The current status of the Standard Model prediction for the anomalous magnetic moment of the muon is briefly reviewed and compared with the present experimental value.

\section{Introduction}

Schwinger's 1948 calculation [1] of the leading QED contribution to the anomalous magnetic moment of the muon $a_{\mu}=\left(g_{\mu}-2\right) / 2$, equal to the one of the electron, was one of the very first results of this theory, and one of its early confirmations. During the last few years, in a sequence of increasingly precise measurements, the E821 Collaboration at Brookhaven has determined $a_{\mu}$ with a fabulous relative precision of 0.5 parts per million (ppm) 2 234, serving as an invaluable tool to test all sectors of the Standard Model (SM) and to scrutinize viable alternatives to this theory [5]. This note provides a brief summary of the present status of the three contributions into which the SM prediction $a_{\mu}^{S M}$ is usually split - QED, electroweak and hadronic - and a comparison with the current experimental value.

\section{QED and Electroweak Contributions}

The QED contribution to $a_{\mu}$ arises from the subset of SM diagrams containing only leptons $(e, \mu, \tau)$ and photons. The lowest-order contribution is $a_{\mu}^{Q E D}(1$ loop $)=\alpha /(2 \pi)$ 1]. Also the two- and three-loop QED terms are known analytically - see [ 6] for an update and a review of these contributions. The four-loop term has been evaluated numerically, a formidable task first accomplished by Kinoshita and his collaborators in the early 1980s [7. The latest analysis appeared in [8]. Note that this four-loop contribution is about six times larger than the present experimental uncertainty of $a_{\mu}$ ! The evaluation of the

\footnotetext{
* This work was supported in part by the European Program MRTN-CT-2004-503369.
}

five-loop QED contribution is in progress 9].

Adding up these terms, using the latest CODATA [10 recommended value for the finestructure constant $\alpha^{-1}=137.03599911(46)$, known to $3.3 \mathrm{ppb}$, one obtains [6] $a_{\mu}^{Q E D}=$ $116584718.8(0.3)(0.4) \times 10^{-11}$. The first error is mainly due to the uncertainty of the $O\left(\alpha^{5}\right)$ term, while the second one is caused by the $3.3 \mathrm{ppb}$ uncertainty of the fine-structure constant.

The electroweak (EW) contribution to $a_{\mu}$ is suppressed by a factor $\left(m_{\mu} / M_{W}\right)^{2}$ with respect to the QED effects. The one-loop part was computed in 1972 by several authors [11: $a_{\mu}^{E W}(1$ loop $)=$ $\frac{5 G_{\mu} m_{\mu}^{2}}{24 \sqrt{2} \pi^{2}}\left[1+\frac{1}{5}\left(1-4 \sin ^{2} \theta_{W}\right)^{2}+O\left(m_{\mu}^{2} / M_{Z, W, H}^{2}\right)\right]$, where $G_{\mu}=1.16637(1) \times 10^{-5} \mathrm{GeV}^{-2}$. Employing the on-shell definition $\sin ^{2} \theta_{W}=1-$ $M_{W}^{2} / M_{Z}^{2}$ [12], where $M_{Z}=91.1875(21) \mathrm{GeV}$ and $M_{W}$ is the SM prediction of the $W$ mass (which can be derived, for example, from the simple formulae of 13] leading to $M_{W}=80.383 \mathrm{GeV}$ for the Higgs mass $M_{H}=150 \mathrm{GeV}$ ), we obtain $a_{\mu}^{E W}(1$ loop $)=194.8 \times 10^{-11}$.

The two-loop EW contribution to $a_{\mu}$ is not negligible because of large factors of $\ln \left(M_{Z, W} / m_{f}\right)$, where $m_{f}$ is a fermion mass scale much smaller than $M_{W}$ [14. It was computed in 1995 15]. The proper treatment of the contribution of the light quarks was addressed in 1617]. These refinements significantly improved the reliability of the fermionic part (that containing closed fermion loops) of $a_{\mu}^{E W}$ (two loop) leading, for $M_{H}=$ $150 \mathrm{GeV}$, to $a_{\mu}^{E W}=154(1)(2) \times 10^{-11}$ [17]. The first error is due to hadronic loop uncertainties, while the second one corresponds to an allowed range of $M_{H} \in[114,250] \mathrm{GeV}$, to the current top mass uncertainty, and to unknown three-loop ef- 
fects. The leading-logarithm three-loop contribution to $a_{\mu}^{E W}$ is extremely small [1718. The results of [19] for the two-loop bosonic part of $a_{\mu}^{E W}$, performed without the large $M_{H}$ approximation previously employed, agree with the previous evaluation [15] in the large Higgs mass limit. Work is also in progress for an independent recalculation based on the numerical methods of [20].

\section{The Hadronic Contribution}

The evaluation of the hadronic leading-order contribution $a_{\mu}^{H L O}$, due to the hadronic vacuum polarization correction to the one-loop diagram, involves long-distance QCD for which perturbation theory cannot be employed. However, using analyticity and unitarity, it was shown long ago that this term can be computed from hadronic $e^{+} e^{-}$annihilation data via the dispersion integral $a_{\mu}^{H L O}=\frac{1}{4 \pi^{3}} \int_{4 m_{\pi}^{2}}^{\infty} d s K(s) \sigma^{(0)}(s)$ 21], where $\sigma^{(0)}(s)$ is the total cross section for $e^{+} e^{-}$annihilation into any hadronic state, with extraneous QED corrections subtracted off. The kernel function $K(s)$ decreases monotonically for increasing $s$.

A prominent role among all $e^{+} e^{-}$annihilation measurements is played by the precise data collected in 1994-95 by the CMD-2 detector at the VEPP-2M collider in Novosibirsk for the $e^{+} e^{-} \rightarrow$ $\pi^{+} \pi^{-}$cross section at values of $\sqrt{s}$ between 0.61 and $0.96 \mathrm{GeV} 22$. The quoted systematic error of these data is $0.6 \%$, dominated by the uncertainties in the radiative corrections $(0.4 \%)$. Recently 23] the CMD-2 Collaboration released its 1996-98 measurements for the same cross section in the full energy range $\sqrt{s} \in[0.37,1.39] \mathrm{GeV}$. The part of these data for $\sqrt{s} \in[0.61,0.96] \mathrm{GeV}$ (quoted systematic error $0.8 \%$ ) agrees with their earlier result published in 22. Also the SND Collaboration (at the VEPP-2M collider as well) recently presented its analysis of the $e^{+} e^{-} \rightarrow \pi^{+} \pi^{-}$ process for $\sqrt{s}$ between 0.39 and $0.98 \mathrm{GeV}$, with a systematic uncertainty of $1.3 \%(3.2 \%)$ for $\sqrt{s}$ larger (smaller) than $0.42 \mathrm{GeV}$ 24]. A hint of discrepancy, at the level of the combined systematic error, occurs between the CMD-2 and SND measurements (the contribution to $a_{\mu}^{H L O}$ of the SND data is a bit higher than the corresponding one from CMD-2) 23. Further significant progress is expected from the new $e^{+} e^{-}$collider VEPP-2000 under construction in Novosibirsk [23].

In 2004 the KLOE experiment at the DA $\Phi N E$ collider in Frascati presented a precise measurement of $\sigma\left(e^{+} e^{-} \rightarrow \pi^{+} \pi^{-}\right)$via the initial-state radiation (ISR) method at the $\phi$ resonance [25]. This cross section was extracted for $\sqrt{s}$ between 0.59 and $0.97 \mathrm{GeV}$ with a systematic error of $1.3 \%$ and a negligible statistical one. There are some discrepancies between the KLOE and CMD-2 results (KLOE's data lying higher than the CMD2 fit below the $\rho$ peak, and lower on the peak and above it), although their integrated contributions to $a_{\mu}^{H L O}$ are similar [23]. The data of KLOE and SND disagree above the $\rho$ peak, where the latter are significantly higher. The study of the $e^{+} e^{-} \rightarrow \pi^{+} \pi^{-}$process via the ISR method is also in progress at BABAR [26] and BELLE [27]. On the theoretical side, analyticity, unitarity and chiral symmetry provide strong constraints for the pion form factor in the low-energy region 28 .

The evaluations of the dispersive integral based on the analysis 22 of the 1994-95 CMD-2 data are in good agreement: ${ }^{1}$

$$
\begin{aligned}
& \text { [31 } a_{\mu}^{H L O}=6934(53)_{\exp }(35)_{\text {rad }} \times 10^{-11} \text {, } \\
& \text { 32] } a_{\mu}^{H L O}=6948(86) \times 10^{-11} \text {, } \\
& 33 \quad a_{\mu}^{H L O}=6924(59)_{\exp }(24)_{\text {rad }} \times 10^{-11} \text {, } \\
& \text { [34] } a_{\mu}^{H L O}=6944(48)_{e x p}(10)_{r a d} \times 10^{-11} \text {. }
\end{aligned}
$$

Reference 31 updates [35] and already includes KLOE's results. The recently released data of CMD-2 23] and SND 24] are not yet included.

The authors of 36 pioneered the idea of using vector spectral functions derived from the study of hadronic $\tau$ decays (see [3738 for recent reviews) to improve the evaluation of the dispersive integral. Indeed, assuming isospin invariance to hold, the isovector part of the cross section for $e^{+} e^{-} \rightarrow$ hadrons can be calculated via the Conserved Vector Current relations from $\tau$-decay spectra. The latest analysis with ALEPH 39, CLEO [40, and OPAL 41] data yields $a_{\mu}^{H L O}=7110(50)_{\exp }(8)_{\operatorname{rad}}(28)_{S U(2)} \times$ $10^{-11}$ 35]. Isospin-breaking corrections were applied [42]. Information from $\tau$ decays was also

${ }^{1}$ The evaluation of [29] is not included as its result is being revised [30. 
included in one of the analyses of 34, leading to $a_{\mu}^{H L O}=7027(47)_{\exp }(10)_{\text {rad }} \times 10^{-11}$.

Although the precise CMD-2 $e^{+} e^{-} \rightarrow \pi^{+} \pi^{-}$ data [22] are consistent with the corresponding $\tau$ ones for energies below $\sim 0.85 \mathrm{GeV}$, they are significantly lower for larger energies. KLOE's $\pi^{+} \pi^{-}$ spectral function confirms this discrepancy with the $\tau$ data; on the contrary, the recent SND results are compatible with them [24]. This discrepancy could be caused by inconsistencies in the $e^{+} e^{-}$or $\tau$ data, or in the isospin-breaking corrections which must be applied to the latter. Indeed, the mentioned disagreements between the $e^{+} e^{-}$data sets need careful consideration. On the other hand, in spite of the agreement of the $\tau$ data sets 38, the question remains whether all possible isospin-breaking effects have been properly taken into account 3143].

The hadronic higher-order contribution can be divided into two parts: $a_{\mu}^{H H O}=a_{\mu}^{H H O}(\mathrm{vp})+$ $a_{\mu}^{H H O}(\mathrm{lbl})$. The first term is the $O\left(\alpha^{3}\right)$ contribution of diagrams containing hadronic vacuum polarization insertions 44. Its latest value is $a_{\mu}^{H H O}(\mathrm{vp})=-97.9(0.9)_{e x p}(0.3)_{\mathrm{rad}} \times 10^{-11}$ [33], obtained using $e^{+} e^{-}$annihilation data; it changes by $\sim-3 \times 10^{-11}$ if hadronic $\tau$-decay data are used instead [45. The second term, also of $O\left(\alpha^{3}\right)$, is the hadronic light-by-light contribution; as it cannot be determined from data, its evaluation relies on specific models. In 2001 the authors of [46] uncovered a sign error in earlier evaluations of its dominating pion-pole part. Their estimate, based also on previous results for the quark and charged-pions loop parts [47], is $a_{\mu}^{H O}(\mathrm{lbl})=$ $80(40) \times 10^{-11}$ 48]. A higher value was obtained in 2003 including short-distance QCD constraints: $a_{\mu}^{H H O}(\mathrm{lbl})=136(25) \times 10^{-11}$ [49. Further independent calculations would provide an important check of this result for $a_{\mu}^{H H O}(\mathrm{lbl})$, a contribution whose uncertainty may become the ultimate limitation of the SM prediction of the muon $g-2$.

\section{Standard Model vs. Measurement}

The first column of Table 1 shows $a_{\mu}^{S M}=a_{\mu}^{Q E D}+$ $a_{\mu}^{E W}+a_{\mu}^{H L O}+a_{\mu}^{H H O}$. The values employed for $a_{\mu}^{H L O}$ are indicated by the reference on the left (34 quotes two values, see Sec. 3); all $a_{\mu}^{S M}$ values were

\begin{tabular}{|c|c|c|c|}
\hline$a_{\mu}^{S M}$ & $\times 10^{11}$ & $\Delta \times 10^{11}$ & $\sigma$ \\
\hline 31 & $116591845(69)$ & $235(91)$ & $2.6\langle 3.0\rangle$ \\
\hline 32 & $116591859(90)$ & $221(108)$ & $2.1\langle 2.5\rangle$ \\
\hline 33 & $116591835(69)$ & $245(91)$ & $2.7\langle 3.1\rangle$ \\
\hline 34 & $116591855(55)$ & $225(81)$ & $2.8\langle 3.2\rangle$ \\
\hline 35 & 116592018 (63) & $62(87)$ & $0.7\langle 1.3\rangle$ \\
\hline 34 & $116591938(54)$ & $142(81)$ & $1.8\langle 2.3\rangle$ \\
\hline
\end{tabular}

Table 1: Standard Model vs. measurement.

derived with $a_{\mu}^{H H O}(\mathrm{lbl})=136(25) \times 10^{-11}$ [49]. Errors were added in quadrature. The present world average experimental value for the muon $g-2$ is $a_{\mu}^{E X P}=116592080(60) \times 10^{-11}(0.5 \mathrm{ppm})[3]$. The differences $\Delta=a_{\mu}^{E X P}-a_{\mu}^{S M}$ are listed in the second column of Table 1, while the numbers of "standard deviations" $(\sigma)$ appear in the third one. Higher discrepancies, shown in angle brackets, are obtained if $a_{\mu}^{H H O}(\mathrm{lbl})=80(40) \times$ $10^{-11}$ [48] is used instead of $136(25) \times 10^{-11}$ [4].

\section{Conclusions}

The discrepancies between recent SM predictions of $a_{\mu}$ and the current experimental value vary in a very wide range, from 0.7 to $3.2 \sigma$, according to the values chosen for the hadronic contributions. In particular, the leading-order hadronic contribution depends on which of the two sets of data, $e^{+} e^{-}$collisions or $\tau$ decays, are employed. The puzzling discrepancy between the $\pi^{+} \pi^{-}$spectral functions from $e^{+} e^{-}$and isospinbreaking-corrected $\tau$ data could be caused by inconsistencies in the $e^{+} e^{-}$or $\tau$ data, or in the isospin-breaking corrections applied to the latter. Indeed, disagreements occur between $e^{+} e^{-}$data sets, requiring further detailed investigations. On the other hand, $\tau$ data sets are in agreement, but their connection with the leading hadronic contribution to $a_{\mu}$ is less direct, and one wonders whether all possible isospin-breaking effects have been properly taken into account. Using $e^{+} e^{-}$ data, the SM prediction of the muon $g-2$ deviates from the present experimental value by $2-3 \sigma$.

The impressive results of the E821 experiment are still limited by statistical errors. A new experiment, E969, has been approved (but not yet funded) at Brookhaven in 2004 [450. Its goal is to reduce the present experimental uncertainty by a factor of 2.5 to about $0.2 \mathrm{ppm}$. A letter of 
intent for an even more precise $g-2$ experiment was submitted to J-PARC with the proposal to reach a precision below $0.1 \mathrm{ppm}$ [51]. While the QED and EW contributions appear to be ready to rival these precisions, much effort will be needed to reduce the hadronic uncertainty by a factor of two. This effort is challenging but possible, and certainly well motivated by the excellent opportunity the muon $g-2$ is providing us to unveil (or constrain) "new physics" effects.

Acknowledgments. I would like to thank the organizers for their kind invitation and excellent organization of this workshop, and S. Eidelman and B.L. Roberts for very useful communications.

\section{REFERENCES}

1. J.S. Schwinger, Phys. Rev. 73 (1948) 416.

2. H.N. Brown et al., Phys.Rev.D62 (2000) 91101; Phys.Rev.Lett.86(2001)2227;G.W.Bennett et al., ibid.89 (2002) 101804; ibid.89 (2002) 129903(E).

3. G.W. Bennett et al., Phys. Rev. Lett. 92 (2004) 161802.

4. B.L. Roberts, these proceedings.

5. A. Czarnecki, W.J. Marciano, Phys. Rev. D 64 (2001) 013014.

6. M. Passera, J. Phys. G 31 (2005) R75.

7. T. Kinoshita, W.B. Lindquist, Phys.Rev.Lett.47 (1981)1573; T.Kinoshita et al., ibid.52(1984)717.

8. T.Kinoshita, M.Nio, Phys.Rev.D70(2004)113001.

9. A.L.Kataev, these proceedings hep-ph/0507174; T.Kinoshita,Nucl.Phys.Proc.Suppl.144(2005)206.

10. P.J.Mohr, B.N.Taylor, Rev.Mod.Phys.77(2005)1.

11. R.Jackiw, S.Weinberg, Phys.Rev.D5 (1972) 2396; I. Bars, M. Yoshimura, Phys. Rev. D6 (1972) 374; G. Altarelli et al., Phys. Lett. B40 (1972) 415; W.A. Bardeen et al., Nucl. Phys. B46 (1972) 319; K. Fujikawa et al., Phys. Rev. D 6 (1972) 2923.

12. A. Sirlin, Phys. Rev. D 22 (1980) 971.

13. A. Ferroglia et al., Phys.Rev.D65 (2002) 113002; G. Degrassi, P. Gambino, Nucl.Phys.B567 (2000) 3; G. Degrassi et al., Phys.Lett.B418 (1998) 209.

14. T.V. Kukhto et al., Nucl. Phys. B371 (1992) 567.

15. A. Czarnecki et al., Phys. Rev. D 52 (1995) 2619; Phys. Rev. Lett. 76 (1996) 3267.

16. S. Peris et al., Phys. Lett. B 355 (1995) 523; M. Knecht et al., JHEP 0211 (2002) 003.

17. A. Czarnecki et al., Phys.Rev.D67 (2003) 073006.

18. G. Degrassi, G.F. Giudice, Phys. Rev. D 58 (1998) 053007.

19. S.Heinemeyer et al.,Nucl.Phys.B699(2004)103;T. Gribouk,A.Czarnecki,Phys.Rev.D72(2005)53016.
20. F.V. Tkachov, Nucl. Instrum. Meth. A389 (1997) 309; G. Passarino, Nucl. Phys. B 619 (2001) 257; G. Passarino, S. Uccirati, ibid. 629 (2002) 97; A. Ferroglia et al., ibid. 650 (2003) 162; ibid. 680 (2004) 199; Nucl. Instrum. Meth. A 502 (2003) 391; S. Actis et al., Nucl. Phys. B 703 (2004) 3.

21. C. Bouchiat, L. Michel, J. Phys. Radium 22 (1961) 121; M. Gourdin, E. de Rafael, Nucl. Phys. B 10 (1969) 667.

22. R.R.Akhmetshinet al.,Phys.Lett.B578(2004)285.

23. I. Logashenko, S. Eidelman, Talks at HEP 2005 (Lisbon, Portugal, July 21st-27th 2005).

24. M.N. Achasov et al., hep-ex/0506076

25. A. Aloisio et al., Phys. Lett. B 606 (2005) 12; Nucl. Phys. Proc. Suppl. 144 (2005) 231.

26. M.Davier, Nucl.Phys.Proc.Suppl.144 (2005) 238.

27. B. A. Shwartz, Nucl. Phys. Proc. Suppl. 144 (2005) 245.

28. G. Colangelo, Nucl. Phys. Proc. Suppl. 131 (2004) 185, and references therein.

29. V.V. Ezhela et al., hep-ph/0312114

30. O.V. Zenin, private communication.

31. A. Höcker, hep-ph/0410081

32. F. Jegerlehner, Nucl. Phys. Proc. Suppl. 126 (2004) 325; ibid. 131 (2004) 213.

33. K. Hagiwara et al., Phys.Rev.D69 (2004) 093003.

34. J.F. de Trocóniz, F.J. Ynduráin, Phys. Rev. D 71 (2005) 073008.

35. M. Davier et al., Eur. Phys. J. C 31 (2003) 503.

36. R. Alemany et al., Eur. Phys. J. C 2 (1998) 123.

37. J. Portoles, Nucl.Phys.Proc.Suppl. 144 (2005) 3.

38. M. Davier et al., hep-ph/0507078

39. S. Schael et al., hep-ex/0506072

40. S. Anderson et al., Phys.Rev.D61 (2000) 112002.

41. K. Ackerstaff et al., Eur. Phys. J. C 7 (1999) 571.

42. W.J. Marciano, A. Sirlin, Phys. Rev. Lett. 61 (1988) 1815; V. Cirigliano et al., Phys. Lett. B 513 (2001) 361; JHEP 0208 (2002) 002.

43. S. Ghozzi, F. Jegerlehner, Phys. Lett. B 583, 222 (2004); M. Davier, Nucl. Phys. Proc. Suppl. 131 (2004) 123; K. Maltman, hep-ph/0504201 K. Maltman and C.E. Wolfe, hep-ph/0509224

44. B. Krause, Phys. Lett. B 390 (1997) 392.

45. M. Davier, W.J. Marciano, Annu. Rev. Nucl. Part. Sci. 54 (2004) 115.

46. M.Knecht,A.Nyffeler,Phys.Rev.D65(2002)73034; M.Knecht et al., Phys.Rev.Lett.88 (2002) 71802.

47. M.Hayakawa, T.Kinoshita, Phys.Rev.D57(1998) 465; ib. D66 (2002) 019902(E); J. Bijnens et al., Nucl.Phys.B474 (1996) 379; ib. B626 (2002) 410.

48. A. Nyffeler, Acta Phys. Polon. B 34 (2003) 5197.

49. K. Melnikov, A. Vainshtein, Phys. Rev. D 70 (2004) 113006.

50. R.M. Carey et al., Proposal of the BNL Experiment E969, 2004; B.L. Roberts, hep-ex/0501012

51. J-PARC L.o.I. L17, B.L. Roberts contact person. 